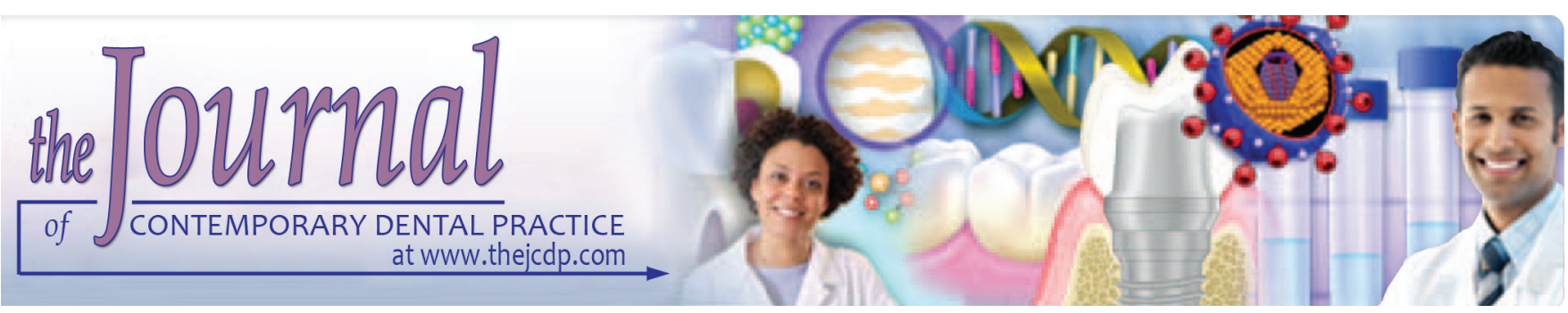

\title{
Comparative Study of Piezoelectric and Rotary Osteotomy Technique for Third Molar Impaction
}

\author{
${ }^{1}$ Sulphi A Basheer, ${ }^{2} \mathrm{R}$ Jay Govind, ${ }^{3}$ Augustine Daniel, ${ }^{4}$ George Sam, ${ }^{5} \mathrm{VJ}$ Adarsh, ${ }^{6}$ Akshatha Rao
}

\begin{abstract}
Introduction: Bone removal is necessary for extracting the third molars that are erupted, partially erupted, and/or impacted in bone. Hence, it is necessary to choose a surgical method or instruments that conform to anatomic landmarks and are based on physiological principles. Many authors have reported injuries to the adjacent tooth, especially the distal part of periodontium after removal of second molar. Hence, the present study was undertaken to assess and compare the surgical and postsurgical outcomes of third molar removal using piezoelectric surgery and rotary bur.
\end{abstract}

Materials and methods: A total of 30 healthy adult individuals who were in need of prophylactic removal of impacted mandibular third molar tooth with ideal condition were included for the study. Individuals were divided randomly into study groups of 15 each, so that the difficulty of surgery will be the same in both the groups. Group I - piezoelectric osteotomy technique and group II - rotary osteotomy technique. The rotary device consists of a hand piece and a rotary speed ranging around $35,000 \mathrm{rpm}$ was used. The piezoelectric device consists of a

\footnotetext{
${ }^{1}$ Department of Dentistry/Oral and Maxillofacial Surgery Sree Gokulam Medical College and Research Foundation Thiruvananthapuram, Kerala, India

${ }^{2}$ Department of Orthodontics and Dentofacial Orthopaedics, PMS College of Dental Science \& Research, Thiruvananthapuram Kerala, India

${ }^{3}$ Department of Oral and Maxillofacial Surgery, Al-Azhar Dental College, Thodupuzha, Kerala, India

${ }^{4}$ Department of Preventive Dental Sciences, College of Dentistry Prince Sattam bin Abdulaziz University, Al-kharj, Kingdom of Saudi Arabia

${ }^{5}$ Department of Conservative Dentistry and Endodontics, Mahe Institute of Dental Sciences and Hospital, Pallor, Puducherry, India

${ }^{6}$ Department of Oral and Maxillofacial Surgery, Century International Institute of Dental Sciences and Research Centre Poinachi, Kerala, India
}

Corresponding Author: Sulphi A Basheer, Department of Dentistry/Oral and Maxillofacial Surgery, Sree Gokulam Medical College and Research Foundation, Thiruvananthapuram, Kerala India, Phone: +919656362236, e-mail: sulphi@gmail.com hand piece, and a frequency of 25 to $29 \mathrm{kHz}$ with a microvibration of 60 to $200 \mathrm{~mm} / \mathrm{sec}$ was used with a boosted working mode. Data were analyzed using unpaired t-test and qualitative data were analyzed using Fischer's exact test.

Results: The average age of the study subjects in the piezosurgery group and rotary group was $28.40 \pm 2.69$ and 30.06 \pm 3.15 years respectively. The time taken for removal of impacted tooth by rotary bur was less than that by piezoelectric device, which was significant statistically $(p<0.05)$. Until the 4 th postoperative day, severity of pain experienced was more in the rotary group, which was statistically significant $(p<0.005)$. Mouth opening was significantly better in the piezoelectric group as compared with rotary bur until the 7 th postoperative day.

Conclusion: The piezosurgery method reduces postoperative pain, trismus, and swelling. Also, it may play an important role in increasing bone density within the extraction socket and decreasing the amount of bone loss of adjacent tooth in the distal aspect.

Clinical significance: In clinical practice, piezosurgery plays an important role because piezosurgery reduces postoperative pain, trismus, and also swelling.

Keywords: Impacted tooth, Mouth opening, Pain, Piezoelectric osteotomy, Rotary osteotomy.

How to cite this article: Basheer SA, Govind RJ, Daniel A, Sam G, Adarsh VJ, Rao A. Comparative Study of Piezoelectric and Rotary Osteotomy Technique for Third Molar Impaction. J Contemp Dent Pract 2017;18(1):60-64.

Source of support: Nil

Conflict of interest: None

\section{INTRODUCTION}

Impacted third molars are frequently reported problems in clinical practice, with a prevalence of 33 to $58.7 \%$. It has been well documented that impacted third molars, either partial or complete, are associated with several complications, including pericoronitis, regional pain, dentoalveolar abscess, trismus, distal caries on second molar, cysts, tumors, and dental arch crowding. Therefore, 
symptomatic or asymptomatic impacted third molars are often extracted to reduce the above-mentioned clinical symptoms. ${ }^{1}$

Third molar surgical removal is one of the most frequent and delicate therapies among the surgical operations dentists must perform. The high-speed rotary hand piece is the most commonly and widely used instrument for impacted tooth removal. However, recently, the piezosurgery technique has been used to carry out safe and effective bone removal using piezoelectric ultrasonic vibrations. Both the tools are used by oral surgeons for osteotomy and odontotomy during surgical third molar extraction. $^{2}$

The surgical removal of impacted third molars may lead to various postoperative side effects, including pain, swelling, trismus, nerve injury, bleeding, and dry sockets. Different strategies are adopted to reduce these complications, including changing the technique of the osteotomy. ${ }^{3}$

Bone removal is a necessity for extracting third molars whether they are partially erupted or fully impacted. Surgical hand pieces with carbide bur are routinely used to remove bone during the extraction of impacted third molars. According to the morphological analysis of bone, the bur produces irregular surfaces and marginal osteonecrosis as a result of high temperature generated during bone removal. ${ }^{4}$ Horton et $\mathrm{al}^{5}$ introduced the ultrasonic inserts in the surgical removal of alveolar bone. The authors have studied histologically the effect of ultrasonic cutting inserts on alveolar bone and reported that ultrasonic inserts remove bone easily and precisely. In addition, the hemorrhage from surgical sites is minimal and there is improved healing with less postoperative complications. Furthermore, the discomfort level of patients after surgery will be minimal.

Vercellotti et $\mathrm{al}^{6}$ reported that the piezoelectric device (piezosurgery) can be an effective tool for carrying out maxillary sinus surgeries. After these reports, piezosurgery has been widely used as an alternative to rotary instruments.

Piezoelectric surgery techniques have opened up a new age for osteotomy, osteoplasty and exodontia in maxillofacial and oral surgery. As well as being selective, the micrometric cuts possible via these techniques maximize surgical precision, resulting in minimal damage to soft tissue. In addition, the cavitation effect provides maximum intraoperative visibility and a blood-free surgical site. ${ }^{7}$

Piezosurgery is a novel technique that has been introduced as a valuable alternative to overcome the disadvantages associated with the conventional rotating bone-cutting instruments. It is performed by means of a device that uses microvibration at a frequency capable of cutting bone. Its mechanism of action is based on the ability of certain ceramics and crystals to deform when an electric current is passed across them, resulting in microvibration at ultrasonic frequency. A frequency of 25 to $30 \mathrm{KHz}$, from a nitride-hardened or diamond-coated insert, allows for selective cut of bone tissue. ${ }^{8}$

\section{AIM}

The aim of this article is to assess and compare the surgical and postsurgical outcomes of third molar removal using piezoelectric surgery and rotary bur.

\section{MATERIALS AND METHODS}

This clinical research was done on individuals who visited the Department of Dentistry/Oral and Maxillofacial surgery, Sree Gokulam Medical College and Research Foundation.

About 30 healthy adult individuals reporting to the Department of Dentistry, in need of prophylactic removal of the lower third molar impacted tooth with ideal condition were considered with the following criteria.

\section{Inclusion Criteria}

- Healthy individuals above 20 years of age

- Individuals having vertical, mesioangular, horizontal mandibular third molar impactions based on radiographic interpretation.

\section{Exclusion Criteria}

- Individuals with systemic disease that could influence healing

- Individuals who do not provide consent

- Individuals on antibiotics in the past 6 weeks or who require antibiotic prophylaxis before extraction

- Individuals who had acute local infection involving the impacted teeth.

As and when the individuals were reported, the study protocol was explained and written consent taken from the study participants. Individuals were randomly allocated to study groups, so that the difficulty of surgery will be the same in both the groups until 15 individuals for each group is over.

Group I - piezoelectric osteotomy technique

Group II - rotary osteotomy technique

Adequate local anesthesia was administered under strict aseptic conditions. Under group I, the impacted tooth was surgically extracted using piezoelectric osteotomy technique, and under group II the impacted tooth was surgically extracted using the rotator osteotomy technique.

Hand piece and foot switch were the components of the rotary device, and it was attached to the power plug. 


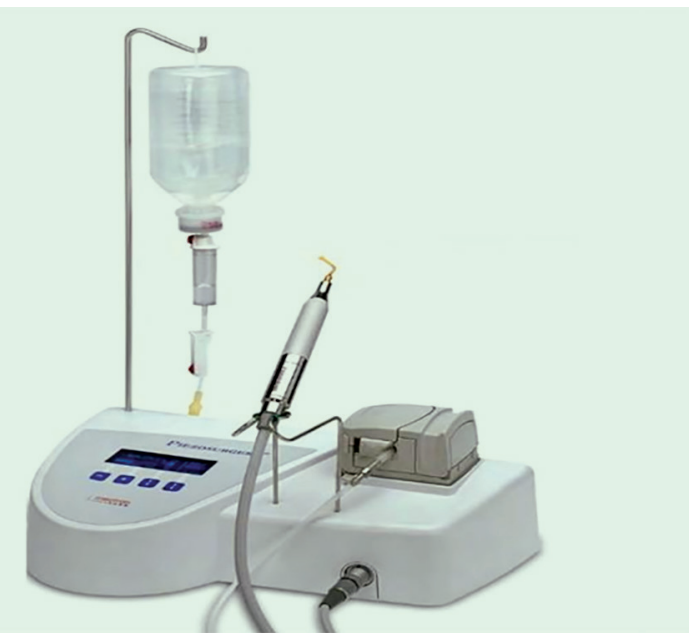

Fig. 1: Piezoelectric device

The rotary speed used ranged around 35,000 rpm. Rotary burs 702 and 703 were used.

The piezoelectric device (Fig. 1) consists of a hand piece and a foot switch, and it was attached to the power plug. A frequency of 25 to $29 \mathrm{kHz}$ with a microvibration of 60 to $200 \mathrm{~mm} / \mathrm{sec}$ was used with a boosted working mode. Piezoelectric burs SL 1, SL 2 and SL 3 were used.

All the individuals underwent surgical removal (Fig. 2) of impacted mandibular third molars under $2 \%$ lignocaine with 1:200,000 adrenaline, with inferior alveolar, lingual, and long buccal nerve blocks administered.

Postoperatively, all individuals received amoxicillin $500 \mathrm{mg}$ tid and diclofenac sodium $50 \mathrm{mg}$ tid for 3 days. Postoperative instructions were given and the sutures were removed on the 7 th day.

The parameters examined in each patient were: time taken for the procedure, patient satisfaction for the procedure, postoperative pain, and postoperative trismus.

Time required for the procedure included starting from the time of bone guttering until the tooth elevation from its socket. Patient satisfaction was assessed subjectively using a graded scale from "very satisfied" to "very unsatisfied."

The degree of pain was recorded for a period of 7 days with reference to predefined values on visual analog scale (VAS). Trismus was evaluated on days 3, 5 and 7 of the postoperative period in millimeters.

Descriptive analysis was done. Results are explained as mean \pm standard deviation (Min - Max) and also as number (\%); $5 \%$ was considered as level of significance with $95 \%$ confidence interval. Quantitative data were analyzed using unpaired t-test and qualitative data were analyzed using Fischer's exact test.

\section{RESULTS}

Table 1 shows comparison of study subjects according to mean age. Mean age of study individuals in the

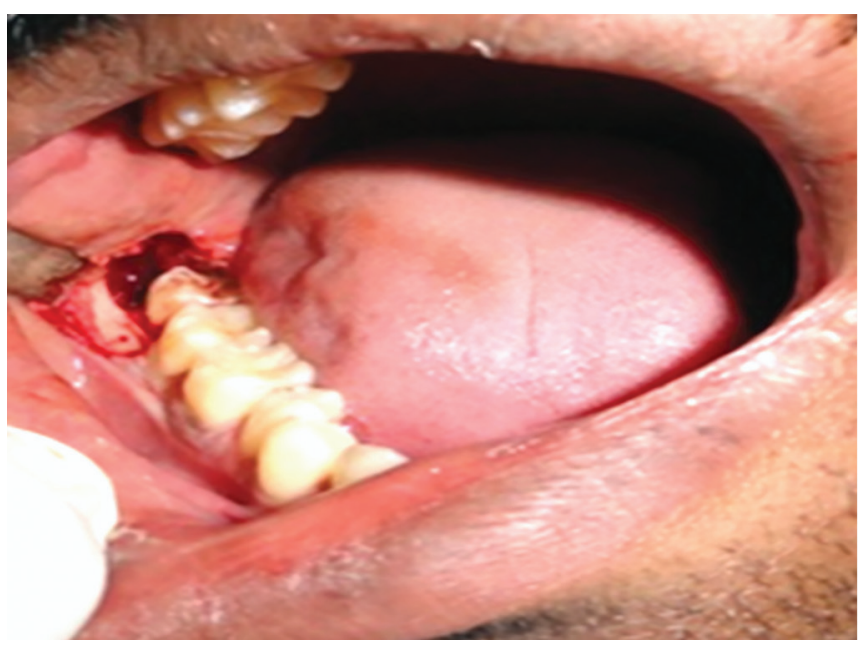

Fig. 2: Surgically removed impacted mandibular third molar

piezosurgery group was $28.40 \pm 2.69$, which was slightly less than the mean age of the rotary group at $30.06 \pm 3.15$. No statistically significant difference was found in mean age between the groups.

Table 2 shows comparison of study individuals based on the type of impaction. There was no statistical difference for the type of impaction among the groups measured using Fischer's exact test.

The time taken for removal of impacted tooth using the piezoelectric device was $48.20 \pm 15.39$ and for rotary bur, it was $34.33 \pm 11.31$, which is less than for the piezoelectric device. Statistically significant difference was found for time taken for the procedure between the groups (Table 3).

Patient satisfaction was evaluated using a grading scale. The results for satisfaction of the procedure were

Table 1: Comparison of mean age among the study groups

\begin{tabular}{llllll}
\hline Groups & $n$ & Mean & $\begin{array}{l}\text { Std. } \\
\text { deviation }\end{array}$ & t-value & $\begin{array}{l}p \text {-value and } \\
\text { significance }\end{array}$ \\
\hline Piezosurgery & 15 & 28.40 & 2.69 & 1.557 & $0.131 \mathrm{NS}$ \\
Rotary & 15 & 30.06 & 3.15 & & \\
\hline P &
\end{tabular}

$p>0.05$, NS: Nonsignificant

Table 2: Type of impaction

\begin{tabular}{llll}
\hline $\begin{array}{l}\text { Type of } \\
\text { impaction }\end{array}$ & Piezosurgery & Rotary & $\begin{array}{l}\text { Fischer's } \\
\text { exact test }\end{array}$ \\
\hline Vertical & 7 & 9 & $\chi^{2}=0.650$ \\
Mesioangular & 6 & 4 & $\mathrm{p}=0.723 \mathrm{NS}$ \\
Horizontal & 2 & 2 & \\
\hline Total & 15 & 15 &
\end{tabular}

$p>0.05$, NS: Nonsignificant

Table 3: Comparison of time taken for the procedure

\begin{tabular}{lllll}
\hline Groups & Mean & $\begin{array}{l}\text { Std. } \\
\text { Deviation }\end{array}$ & $t$-value & $\begin{array}{l}p \text {-value and } \\
\text { significance }\end{array}$ \\
\hline Piezosurgery & 48.20 & 15.39 & 2.811 & $0.009 \mathrm{~S}$ \\
Rotary & 34.33 & 11.31 & & \\
\hline
\end{tabular}

$p<0.05$, S: significant 
Table 4: Comparison of patient's satisfaction of the procedure

\begin{tabular}{llll}
\hline $\begin{array}{l}\text { Patient } \\
\text { satisfaction grade }\end{array}$ & $\begin{array}{l}\text { Piezoelectric } \\
(n=15) \%\end{array}$ & $\begin{array}{l}\text { Rotary } \\
(n=15) \%\end{array}$ & $\begin{array}{l}\text { Fischer's } \\
\text { exact test }\end{array}$ \\
\hline Very satisfied & $6(30.0)$ & $8(40.0)$ & $\chi^{2}=1.385$ \\
Fairly satisfied & $6(40.0)$ & $5(25.0)$ & $\mathrm{p}=0.435 \mathrm{NS}$ \\
Fairly unsatisfied & $3(30.0)$ & $2(35.0)$ & \\
very unsatisfied & 0 & 0 & \\
\hline
\end{tabular}

$p>0.05$, NS: Nonsignificant

almost similar in both the groups, and were without any statistical significant difference (Table 4).

Table 5 reveals the severity of pain as recorded using VAS score, which showed no statistical difference between the piezoelectric and rotary bur on day 1 . However, the number of subjects with severe pain was more in rotary group. Further, until the 4th day, severity of pain experienced was more in rotary group, which was statistically significant $(\mathrm{p}<005)$. On days 5,6 and 7, no statistically significant difference was found among the two groups $(\mathrm{p}<0.05)$.

Mouth opening was measured in millimeters, which showed a statistically significant difference $(p<0.01)$ measured on the 3rd day, 5th day and 7th day between the piezoelectric and rotary bur groups. Mouth opening was significantly better in the piezoelectric group as compared with the rotary bur group until the 7th postoperative day (Table 6).

\section{DISCUSSION}

The important aspect in removing the impacted third molar is to maintain the integrity of the adjacent tooth. The surgical methods may create an exposure of roots and pulpal necrosis. ${ }^{9}$ Hence, it is better to choose a precise technique to remove the tooth without hampering the adjacent tooth. Many studies show that after removal of the mandibular third molar, there will be an injury to the distal aspect of the second molar, and it is still indicated as successful treatment. ${ }^{10-12}$

Recently, piezosurgery has been invented to perform safe and precise surgeries. ${ }^{13}$ To standardize our results, it was conducted on 30 male individuals having their age ranging from 25 to 33 years, in order to remove the gender factor that may play a role in postoperative complications due to hormonal changes that may occur in females.

There was no dropout from the selected sample, and this may be attributed to the well-educated level of the selected individuals and their commitment to their treatment. In addition, the availability of social media makes the follow-up communication with the individuals easier. The duration of the procedure in each site was calculated in terms of minutes starting from the establishment of the flap until the end of suturing. The piezosurgery took a longer time as compared with the control site. This is similar to the research performed by Goyal et al. ${ }^{14}$

Stacchi et al found a limited decrease of implant stability quotient values with piezoelectric methods that increased stability patterns compared with traditional drilling techniques. Not only does the piezoelectric method improve short-term wound healing, other studies have also indicated that it provides the benefit of significantly reduced pain following mastoidectomy. These results, in combination with ours, highlight the unique benefits of piezoelectric devices as safe and minimally invasive tools. ${ }^{11,15}$

Compared with surgery using rotary techniques, piezosurgery was more time consuming due to the slow micrometric cutting action. Surgery time using the ultrasonic osteotomy tended to be shorter as the surgeons accumulated more experience. ${ }^{16}$ Therefore, although the piezoelectric technique is associated with longer surgery time, we believe that with increased experience and the

Table 5: Evaluation of pain (VAS) between the study groups

\begin{tabular}{llllllll}
\hline Duration and groups & No pain & Slight pain & Mild pain & Severe pain & Very severe pain & Fischer's exact test \\
\hline Day 1 & Piezoelectric & 0 & 2 & 6 & 5 & 2 & $\chi^{2}=5.810$ \\
& Rotary & 0 & 0 & 2 & 9 & 4 & $\mathrm{p}=0.121 \mathrm{NS}$ \\
Day 2 & Piezoelectric & 0 & 5 & 8 & 2 & 0 & $\chi^{2}=12.788$ \\
& Rotary & 0 & 0 & 4 & 9 & 2 & $\mathrm{p}=0.005 \mathrm{~S}$ \\
Day 3 & Piezoelectric & 0 & 8 & 7 & 0 & 0 & $\chi^{2}=11.091$ \\
& Rotary & 0 & 3 & 4 & 7 & 1 & $\mathrm{p}=0.011 \mathrm{~S}$ \\
Day 4 & Piezoelectric & 5 & 7 & 3 & 0 & 0 & $\chi^{2}=8.085$ \\
& Rotary & 1 & 4 & 7 & 3 & 0 & $\mathrm{p}=0.044 \mathrm{~S}$ \\
Day 5 & Piezoelectric & 10 & 4 & 1 & 0 & 0 & $\chi^{2}=5.238$ \\
& Rotary & 4 & 8 & 2 & 1 & 0 & $\mathrm{p}=0.155 \mathrm{NS}$ \\
Day 6 & Piezoelectric & 12 & 3 & 0 & 0 & 0 & $\chi^{2}=3.300$ \\
& Rotary & 8 & 5 & 2 & 0 & 0 & $\mathrm{p}=0.192 \mathrm{NS}$ \\
& Piezoelectric & 13 & 2 & 0 & 0 & 0 & $\mathrm{p}$ \\
& Rotary & 10 & 5 & 0 & 0 & $0.390 \mathrm{NS}$ \\
\hline
\end{tabular}

S: Significant; NS: Nonsignificant 
Table 6: Comparison of mean score mouth opening for procedure in the two groups

\begin{tabular}{llllll}
\hline Duration & Groups & Mean & $\begin{array}{l}\text { Std. } \\
\text { Deviation }\end{array}$ & t-value & $\begin{array}{c}p \text {-value and } \\
\text { significance }\end{array}$ \\
\hline Pre-op & Piezoelectric & 35.53 & 1.64 & 0.913 & $0.369 \mathrm{NS}$ \\
& Rotary & 34.93 & 1.94 & & \\
Day 3 & Piezoelectric & 32.80 & 1.37 & 6.634 & $<0.001 \mathrm{HS}$ \\
& Rotary & 28.93 & 1.79 & & \\
Day 5 & Piezoelectric & 33.60 & 1.68 & 8.702 & $<0.001 \mathrm{HS}$ \\
& Rotary & 29.13 & 1.06 & & \\
Day 7 & Piezoelectric & 34.26 & 1.22 & 4.603 & $<0.001 \mathrm{HS}$ \\
& Rotary & 32.40 & 0.98 & & \\
\hline
\end{tabular}

p > 0.05, HS: Highly significant; NS: Nonsignificant

improvement of the technique, piezosurgery will witness reduced surgery time.

In the present study, the pain score was recorded, and it was significantly lesser in the piezoelectric surgery site than while using the rotary instrument. This finding is parallel to the results obtained by Goyal et al, ${ }^{14}$ Mantovani et al, ${ }_{17}^{17}$ and Piersanti et al. ${ }^{13}$ They reported in their studies a significant difference in pain score using the same scale, and all agreed that the site where the impacted mandibular third molar resides, using piezosurgery has less postoperative pain.

These results run along the same line of the findings of a meta-analysis study conducted by Jiang et $\mathrm{al}^{1}$ where seven studies were included in their analysis. The main principle of the study was to make a comparison between piezosurgery and rotary osteotomy techniques. Their meta-analysis shows that although individuals experienced a longer time in piezosurgery, they had less postoperative swelling. Additionally, it is a better alternative technique for removal of impacted mandibular molar.

\section{CONCLUSION}

Within the limitations, the present study concluded that piezosurgery reduces postoperative pain, trismus and swelling. Also, it may play an important role in increasing bone density within the extraction socket and decrease the amount of bone loss in the adjacent mandibular second molar. Compared with conventional rotary technique, piezosurgery has minimal postoperative complications.

\section{REFERENCES}

1. Jiang Q, Qiu Y, Yang C, Yang J, Chen M, Zhang Z. Piezoelectric versus conventional rotary techniques for impacted third molar extraction: a meta-analysis of randomized controlled trials. Medicine 2015 Oct;94(41):1-7.

2. Bartuli FN, Luciani F, Caddeo F, De Chiara L, Di Dio M, Piva P, Ottria L, Arcuri C. Piezosurgery vs high speed rotary handpiece: a comparison between the two techniques in the impacted third molar surgery. Oral Implantol 2013 Jan-Mar;6(1):5-10.

3. Eshghpour M, Nejat AH. Dry socket following surgical removal of impacted third molar in an Iranian population: incidence and risk factors. Niger J Clin Pract 2013 Oct-Dec;16(4):496-500.

4. Kerawala CJ, Martin IC, Allan W, Williams ED. The effects of operator technique and bur design on temperature during osseous preparation for osteosynthesis self-tapping screws. Oral Surg Oral Med Oral Pathol Oral Radiol Endod 1999 Aug;88(2):145-150.

5. Horton JE, Tarpley TM Jr, Jacoway JR. Clinical applications of ultrasonic instrumentation in the surgical removal of bone. Oral Surg Oral Med Oral Pathol 1981 Mar;51(3):236-242.

6. Vercellotti T, De Paoli S, Nevins M. The piezoelectric bony window osteotomy and sinus membrane elevation: introduction of a new technique for simplification of the sinus augmentation procedure. Int J Periodontics Restorative Dent 2001 Dec;21(6):561-567.

7. Gopal I. Comparison of a piezoelectric and a standard surgical handpiece in third molar surgery. 2010 Jan.

8. Arakji H, Shokry M, Aboelsaad N. Comparison of piezosurgery and conventional rotary instruments for removal of impacted mandibular third molars: a randomized controlled clinical and radiographic trial. Int J Dent 2016;2016:1-7.

9. Chang HH, Lee JJ, Kok SH, Yang PJ. Periodontal healing after mandibular third molar surgery - a comparison of distolingual alveolectomy and tooth division techniques. Int J Oral Maxillofac Surg 2004 Jan;33(1):32-37.

10. Kugelberg CF, Ahlstrom U, Ericson S, Hugoson A. Periodontal healing after impacted lower third molar surgery. A retrospective study. Int J Oral Surg 1985 Feb;14(1):29-40.

11. Tsai S-J, Chen Y-L, Chang H-H, Shyu Y-C, Lin C-P. Effect of piezoelectric instruments on healing propensity of alveolar sockets following mandibular third molar extraction. J Dent Sci 2012 Sep;7(3):296-300.

12. Kugelberg CF, Ahlstrom U, Ericson S, Hugoson A, Kvint S. Periodontal healing after impacted lower third molar surgery in adolescents and adults. A prospective study. Int J Oral Maxillofac Surg 1991 Feb;20(1):18-24.

13. Piersanti L, Dilorenzo M, Monaco G, MarchettiC. Piezosurgery or conventional rotatory instruments for inferior third molar extractions? J Oral Maxillofac Surg 2014 Sep;72(9):1647-1652.

14. Goyal M, Marya K, Jhamb A, Chawla S, Sonoo PR, Singh V, Aggarwal A. Comparative evaluation of surgical outcome after removal of impacted mandibular third molars using a Piezotome or a conventional handpiece: a prospective study. Br J Oral Maxillofac Surg 2012 Sep;50(6):556-561.

15. Crippa B, Salzano FA, Mora R, Dellepiane M, Salami A, Guastini L. Comparison of postoperative pain: piezoelectric device versus microdrill. Eur Arch Otorhinolaryngol 2011 Sep;268(9):1279-1282.

16. Beziat JL, Bera JC, Lavandier B, Gleizal A. Ultrasonic osteotomy as a new technique in craniomaxillofacial surgery. Int J Oral Maxillofac Surg 2007 Jun;36(6):493-500.

17. Mantovani E, Arduino PG, Schierano G, Ferrero L, Gallesio G, Mozzati M, Russo A, Scully C, Carossa S. A splitmouth randomized clinical trial to evaluate the performance of piezosurgery compared with traditional technique in lower wisdom tooth removal. J Oral Maxillofac Surg 2014 Oct;72(10): 1890-1897. 